\title{
MASSAGEM EM JOVENS COM SINTOMAS DEPRESSIVOS
}

\author{
Denise Correa de Almeida, Larissa Aguiar Azevedo-Graduanda, Felipe Ramos \\ Scholz e André Leonardo da S. Nessi \\ Escola de Ciências da Saúde UAM-SP \\ denisealmeida28@yahoo.com
}

Acesso DOI: http://dx.doi.org/10.34059/ciejop.2019v27i1-13

\section{RESUMO}

A depressão se tornou um problema de grande importância nos dias de hoje e parece estar relacionada a uma reação ao mundo moderno. O Relatório Mundial de Saúde expõe que a urbanização, o envelhecimento e as mudanças globalizadas nos estilos de vida combinam-se entre si para tornar as doenças crônicas e não transmissíveis - incluindo a depressão - causas cada vez mais importantes de morbidade e de mortalidade. Objetivo desta pesquisa é verificar os benefícios da massagem em jovens com sintomas depressivos. Metodologia: será desenvolvida uma pesquisa quantitativa e exploratória, através de levantamentos de dados referenciais. E serão utilizados os critérios para inclusão como resumos dos artigos e livros sobre técnicas de massagem na cabeça, face e corpo aplicados em jovens com sintomas de depressão, entre os anos de 2001 a 2019, com análise dos dados comparativos e percentual. Resultados: nesta pesquisa foram encontrados cento e quarenta artigos, sendo selecionado apenas vinte e dois utilizando as ferramentas de pesquisa Pubmed, Lilacs, Google Acadêmico, Bireme e Scielo. Conclusão: Tendo em vista os aspectos observados pode se analisar sobre os efeitos fisiológicos, emocionais e bioenergéticos causados em pessoas com sintomas depressivos, sendo abordadas massagens que proporcionam efeitos benéficos sobre a depressão e correlacionando esses efeitos com o sistema nervoso central. Visto então, que há liberação de endorfinas mediante as massagens diminuindo o quadro depressivo.

Palavras-chave: Massagem, jovens, sintomas depressivos.

\begin{abstract}
Depression has become a problem of great importance these days and seems to be related to a reaction to the modern world. The World Health Report shows that urbanization, aging and globalized lifestyle changes combine to make chronic and non-communicable diseases - including depression - increasingly important causes of morbidity and mortality. Objective of this research is to verify the benefits of massage in young people with depressive symptoms. Methodology: a quantitative and exploratory research will be developed, through surveys of reference data. And the criteria for inclusion as summaries of articles and books on head, face and body massage techniques applied to young people with symptoms of depression will be used between the years 2001 and 2019, with analysis of the comparative and percentage data. Results: in this research were found one hundred and forty articles, being selected only twenty-two using the research tools Pubmed, Lilacs, Google Scholar, Bireme and Scielo. Conclusion: In view of the observed aspects, the physiological, emotional and bioenergetic effects caused in people with depressive
\end{abstract}


symptoms can be analyzed, with massages that offer beneficial effects on depression and correlate these effects with the central nervous system. Seen then, that there is release of endorphins through the massages decreasing the depressive picture.

Keywords: Massage, young, depressive symptoms.

\section{INTRODUÇÃO}

A depressão é a consequência de um desequilíbrio bioquímico no cérebro. Os Neurotransmissores param de se comunicar, reduzindo a Serotonina e Noradrenalina, causando em pessoas, sintomas da depressão. Experiências traumáticas, uso excessivo de psicotrópicos, elitismo e melancolia também são fatores que desencadeiam a Depressão (Ferreira et al., 2015).

A depressão pode provocar no indivíduo, em um período mínimo de duas semanas ou mais, sintomas depressivos que podem impactar substancialmente em suas relações sociais, como por exemplo, o ambiente de trabalho, familiar e nos estudos (Régis et al., 2016).

As pessoas em uma maneira geral se alegram e se entristecem com facilidade, por acontecimentos da vida. No entanto, em algumas pessoas essas oscilações ocorridas são mais prolongadas provocando problemas, como a depressão. Assim sendo a depressão a doença do século, estimando se que seja provocada por falhas ocorridas nos neurotransmissores que são dirigentes pela produção dos hormônios como endorfina, dopamina, serotonina e noradrenalina, e também pode ocorrer a diminuição na liberação de neurotransmissores fazendo com que o sistema nervoso central funcione com menos neurotransmissores (Andrade, 2003).

Segundo vários autores, existem vários sintomas que caracterizam a Depressão, entre eles: afeta o humor, melancolia profunda, aperto no peito, sensação de vazio, alteração no sono (insônia ou excesso de sono), falta ou excesso de apetite, dificuldade de concentração, falta de energia, pensamento de morte, sentimento de culpa, tristeza profunda ou tentativa de suicídio (Antunes e Monico, 2015; Ferreira et al,2015; Fonseca et al, 2008; Régis et al, 2016).

A Organização Mundial da Saúde - OMS estima que em 2030 a depressão possa se tornar a doença mais frequente do mundo (Laranjeira, 2015).

Para a OMS (2019), podem ser considerados jovens ou o uso dos termos jovens adultos para englobar a faixa etária de 20 a 24 anos de idade (young adults). 
Os gêneros masculinos e femininos, segundo os estados da depressão, mostram que a mulher é mais propensa a ter sintomas de depressão do que o homem, pois resulta de fatores biológicos, como fatores genéticos e hormonais (Coutinho et al, 2015).

Uma pesquisa realizada na cidade de Florianópolis com 1.560 jovens relata uma prevalência de $9 \%$ dos sintomas depressivos em indivíduos de ambos os sexos com faixa etária entre 20 a 29 anos. Os dados relatados sugerem que nesta fase etária, a juventude adulta, possui um fator de risco relacionado ao contexto sócio cultural que parte dos jovens pesquisados, ressalta que, fatores como uma alimentação inadequada, consumo frequente de álcool, entrada no mercado de trabalho em paralelo com a vida acadêmica contribuem para esta realidade (Régis et al, 2016).

A Massoterapia é a arte científica e o sistema de aplicar manual e sistematicamente uma técnica ao tecido mole superficial da pele, músculos, tendões, ligamentos e às fáscias, e o toque é uma forma de comunicação fundamental e personalizada, possibilitando respostas fisiológicas como as mudanças na concentração de hormônios, alteração na atividade dos sistemas nervosos centrais e periféricos e na regulação dos ritmos do corpo (Fritz, 2002).

A massagem é uma ferramenta terapêutica para o alívio das dores e prevenções de várias doenças. Um de seus objetivos é gerar autonomia em seus pacientes para que ele possa ter mais consciência corporal. Normalmente, quem procura a Massagem, são pessoas que estão sentindo dores físicas, sejam elas por algum tipo de esforço ou por uma carga psicossomática (Cassar, 2001).

Pode se identificar benefícios das massagens orientais e ocidentais, em sintomas depressivos de acordo com algumas referências: Gera o aumento da confiança por melhorar a percepção corporal, causando uma sensação de acolhimento e bem-estar. Possui efeito calmante, o toque terapêutico diminui a ansiedade aguda em pacientes hospitalizados em procedimentos pós-cirúrgicos. (Seubert e Veronese, 2008).

Diminui a exaustão, ajudando no alivio dos efeitos do estresse, hipertensão, úlceras, indigestão, doenças infecciosas, distúrbios gastrointestinais, insônia, dores de cabeça, ansiedade e depressão; O toque estimula o cérebro a produção de endorfinas (hormônio produzido pela glândula hipófise, que possui efeito analgésico) pela glândula pituitária. (Seubert e Veronese, 2008).

Fórum Internacional de Qualidade de Vida e Saúde - Curitiba, 15 de junho de 2019 


\section{OBJETIVO}

Objetivo desta pesquisa é verificar os benefícios da massagem em jovens com sintomas depressivos.

\section{METODOLOGIA}

Será desenvolvida uma pesquisa quantitativa e exploratória, através de levantamentos de dados referenciais em revistas, livros e publicações científicas. Onde serão utilizadas as ferramentas de pesquisa Pubmed, Lilacs, Google Acadêmico, Bireme e Scielo.

Os critérios para inclusão são resumos dos artigos e livros sobre técnicas de massagem na cabeça, face e corpo aplicados em jovens com sintomas de depressão, sendo selecionados trinta artigos ou livros, entre os anos de 2001 a 2019, com análise dos dados comparativos e percentual.

Como objetivos secundários, serão apreciados os resultados além dos benefícios fisiológicos, os efeitos emocionais de bem-estar e respostas bioenergéticas.

\section{RESULTADOS E DISCUSSÃO}

Dos duzentos artigos pesquisados para revisão apenas trinta foram selecionados, sendo setorizados em efeitos fisiológicos, emocionais e bioenergéticos.

Quadro 1 - quadro quantitativo exploratório de artigos sobre os efeitos das técnicas de massagem

\begin{tabular}{|c|c|c|c|c|}
\hline AUTOR & ANO & MASSAGEM & EFEITOS & PARA DEPRESSÃO \\
\hline CASSAR & 2001 & $\begin{array}{l}\text { Massagem } \\
\text { terapêutica }\end{array}$ & $\begin{array}{l}\text { Relaxamento do músculo; } \\
\text { estimulação do sistema } \\
\text { nervoso; melhora a } \\
\text { produção de secreções } \\
\text { glandulares; da circulação } \\
\text { sanguínea; ativar o eixo } \\
\text { hipotálamo e hipófise; } \\
\text { melhora do estado de } \\
\text { ansiedade, medo, raiva e } \\
\text { depressão; }\end{array}$ & $\begin{array}{l}\text { Estimulação do } \\
\text { sistema nervoso; } \\
\text { ativar o eixo } \\
\text { hipotálamo e } \\
\text { hipófise; melhora do } \\
\text { estado de } \\
\text { ansiedade, medo, } \\
\text { raiva e depressão; }\end{array}$ \\
\hline BERTOJA & 2007 & Massagem Relaxante & $\begin{array}{l}\text { Relaxamento; alívio do } \\
\text { estresse; aumento da } \\
\text { circulação venosa e } \\
\text { arterial; das trocas } \\
\text { metabólicas; da histamina; } \\
\text { da melatonina; da }\end{array}$ & $\begin{array}{l}\text { Diminuição da } \\
\text { ansiedade, } \\
\text { depressão, dor; } \\
\text { alívio do estresse; } \\
\text { aumento da } \\
\text { melatonina; da }\end{array}$ \\
\hline
\end{tabular}

Fórum Internacional de Qualidade de Vida e Saúde - Curitiba, 15 de junho de 2019 


\begin{tabular}{|c|c|c|c|c|}
\hline & & & $\begin{array}{l}\text { serotonina e da endorfina; } \\
\text { diminuição da ansiedade, } \\
\text { depressão, dor; melhora } \\
\text { do sistema imunológico; } \\
\text { vasodilatação. }\end{array}$ & $\begin{array}{l}\text { histamina; } \\
\text { serotonina e da } \\
\text { endorfina; melhora } \\
\text { do sistema } \\
\text { imunológico; }\end{array}$ \\
\hline GILLANDERS & 2008 & Reflexologia & $\begin{array}{l}\text { Restauração do equilíbrio; } \\
\text { estimulação do poder } \\
\text { curativo do corpo; melhora } \\
\text { do sistema imunológico, } \\
\text { estimulação do sistema } \\
\text { nervoso central; melhora } \\
\text { da depressão. }\end{array}$ & $\begin{array}{l}\text { Restauração do } \\
\text { equilíbrio; } \\
\text { estimulação do } \\
\text { poder curativo do } \\
\text { corpo; estimulação } \\
\text { do sistema nervoso } \\
\text { central; melhora da } \\
\text { depressão; do } \\
\text { sistema imunológico; }\end{array}$ \\
\hline NESSI & 2010 & $\begin{array}{l}\text { Massagem } \\
\text { Antiestresse }\end{array}$ & $\begin{array}{l}\text { Relaxamento; diminui as } \\
\text { tensões musculares; bem- } \\
\text { estar físico e mental; } \\
\text { aumento da consciência } \\
\text { corporal; melhora a } \\
\text { depressão, ansiedade, } \\
\text { insônia e sintomas } \\
\text { emocionais. }\end{array}$ & $\begin{array}{l}\text { Diminui as tensões } \\
\text { musculares; bem- } \\
\text { estar físico e mental; } \\
\text { aumento da } \\
\text { consciência corporal; } \\
\text { melhora a } \\
\text { depressão, } \\
\text { ansiedade, insônia e } \\
\text { sintomas } \\
\text { emocionais. }\end{array}$ \\
\hline MENEGUZZI & 2011 & Ayurvédica Abhyanga & $\begin{array}{l}\text { Equilíbrio da energia do } \\
\text { corpo; eliminação de } \\
\text { toxinas; redução do } \\
\text { estresse; ansiedade; } \\
\text { melhora a qualidade do } \\
\text { sono; da pele; equilíbrio } \\
\text { físico e metal; } \\
\text { harmonização dos } \\
\text { chakras; redução da } \\
\text { ansiedade e depressão; }\end{array}$ & $\begin{array}{l}\text { Equilíbrio da energia } \\
\text { do corpo; redução } \\
\text { do estresse; } \\
\text { ansiedade; melhora } \\
\text { a qualidade do sono; } \\
\text { equilíbrio físico e } \\
\text { metal; harmonização } \\
\text { dos chakras; } \\
\text { redução da } \\
\text { ansiedade e } \\
\text { depressão; }\end{array}$ \\
\hline GNATTA & 2011 & $\begin{array}{l}\text { Massagem com } \\
\text { Aromaterapia }\end{array}$ & $\begin{array}{l}\text { Equilibra as emoções; } \\
\text { melhorar o bem-estar } \\
\text { físico e mental; ligação } \\
\text { direta com o sistema } \\
\text { nervoso central; estímulo } \\
\text { ao sistema límbico; } \\
\text { melhora o estresse; } \\
\text { melancolia; depressão; } \\
\text { debilidade; raiva; medo; } \\
\text { ciúme; culpa; apatia; } \\
\text { mudança de humor; } \\
\text { confuã̃o e timidez. }\end{array}$ & $\begin{array}{l}\text { Equilibra as } \\
\text { emoções; melhorar o } \\
\text { bem-estar físico e } \\
\text { mental; ligação } \\
\text { direta com o sistema } \\
\text { nervoso central; } \\
\text { melhora o estresse; } \\
\text { melancolia; } \\
\text { depressão; } \\
\text { debilidade; raiva; } \\
\text { medo; ciúme; culpa; } \\
\text { apatia; mudança de } \\
\text { humor; confusão e } \\
\text { timidez. }\end{array}$ \\
\hline RODRIGUES & 2017 & Massagem Ayurveda & $\begin{array}{l}\text { Desbloqueia a energia } \\
\text { vital; melhora tensões; } \\
\text { elimina toxinas; melhora o } \\
\text { sistema imunológico e } \\
\text { circulatório; realinha os } \\
\text { chakras; reequilibra a } \\
\text { energia; relaxamento; } \\
\text { estabiliza o sistema } \\
\text { nervoso central, linfático e } \\
\text { muscular; provoca o } \\
\text { desbloqueio emocional; }\end{array}$ & $\begin{array}{l}\text { Desbloqueia a } \\
\text { energia vital; } \\
\text { melhora tensões; } \\
\text { sistema imunológico; } \\
\text { estabiliza o sistema } \\
\text { nervoso central; } \\
\text { realinha os chakras; } \\
\text { melhora os sintomas } \\
\text { de depressão e } \\
\text { estresse; }\end{array}$ \\
\hline
\end{tabular}

Fórum Internacional de Qualidade de Vida e Saúde - Curitiba, 15 de junho de 2019 


\begin{tabular}{|c|c|c|c|c|}
\hline & & & $\begin{array}{l}\text { melhora os sintomas de } \\
\text { depressão e estresse; }\end{array}$ & \\
\hline CUNICO & 2017 & $\begin{array}{l}\text { Massagem com } \\
\text { Pedras Quentes }\end{array}$ & $\begin{array}{l}\text { Relaxar a musculatura; } \\
\text { equilibrar as energias do } \\
\text { corpo; promove a melhora } \\
\text { no sistema circulatório; } \\
\text { equilíbrio emocional; reduz } \\
\text { dores musculares; diminui } \\
\text { o estresse; tonificação } \\
\text { muscular; tratamento de } \\
\text { depressão, ansiedade; } \\
\text { auxiliam na tonificação } \\
\text { muscular; revigorando a } \\
\text { energia vital; reduzindo } \\
\text { inflamações; estimula o } \\
\text { sistema nervoso central; }\end{array}$ & $\begin{array}{l}\text { Equilibrar as } \\
\text { energias do corpo; } \\
\text { equilíbrio emocional; } \\
\text { reduz dores } \\
\text { musculares; diminui } \\
\text { o estresse; } \\
\text { tratamento de } \\
\text { depressão, } \\
\text { ansiedade; } \\
\text { revigorando a } \\
\text { energia vital; } \\
\text { estimula o sistema } \\
\text { nervoso central; }\end{array}$ \\
\hline ALVES & 2018 & Massagem com Velas & $\begin{array}{l}\text { Diminui a depressão, } \\
\text { estresse, fadiga, insônia e } \\
\text { ansiedade; alivio de } \\
\text { tensões musculares; } \\
\text { melhora da circulação } \\
\text { sanguínea; equilíbrio } \\
\text { energético do corpo; } \\
\text { promove o relaxamento } \\
\text { muscular; bem estar; }\end{array}$ & $\begin{array}{l}\text { Diminui a } \\
\text { depressão, } \\
\text { estresse, fadiga, } \\
\text { insônia e ansiedade; } \\
\text { alivio de tensões } \\
\text { musculares; } \\
\text { equilíbrio energético } \\
\text { do corpo; bem estar; }\end{array}$ \\
\hline
\end{tabular}

Pode se observar no quadro acima que Cassar (2001) cita que os efeitos fisiológicos da massagem terapêutica são promovidos devido a influencia de fatores mecânicos e reflexos que promovem relaxamento, melhora da contratação involuntária dos músculos causados pelo desequilíbrio hidroeletrolítico, melhora da produção de secreção glandular, melhora da circulação sanguínea, linfática, alivio de raiva, depressão, ansiedade e medo. Porém, a massagem pode causar estímulos no sistema nervoso central através dos níveis sistêmicos e periféricos o que faz ativar o eixo hipotálamo e hipófise.

Já Bertoja (2007) salienta que a massagem relaxante quando realizada tem efeitos reflexos e psicológicos no corpo, ou seja, os efeitos fisiológicos só são causados quando se utiliza movimentos com pressão superficial ou profunda que tem mobilização de tecido, tendo o objetivo de estimular os receptores sensitivos para que se leve a informação para o sistema nervoso central fazendo com que desative o sistema simpático, e consequentemente ative 0 parassimpático provocando o relaxamento dos músculos. Portanto, através do sistema parassimpático se obtém efeitos na diminuição da ansiedade, depressão, dor e melhora do sistema imunológico, pois quando a massagem está sendo realizada estimula a vasodilatação liberando os vasos sanguíneos e pode também estimular o eixo hipotálamo e hipófise causando a liberação de hormônios como histamina que Fórum Internacional de Qualidade de Vida e Saúde - Curitiba, 15 de junho de 2019 
promove a defesa do corpo, melatonina que leva ao sono profundo, serotonina e endorfina que ocasionaram sensação de bem-estar.

Entretanto Gillanders (2008) enfatiza que a depressão, o medo, a ansiedade e a dor emocional quando constitui a porção maior das emoções agridem o sistema imunológico, que enfraquece e abre as portas para doenças. Sendo assim, os tratamentos complementares, sobretudo a Reflexologia, restaura o equilíbrio do corpo, melhora o sistema imunológico, estimula o sistema nervoso central e estimula o poder curativo do corpo através da esfera do meridiano, nervos e bioenergético que interfere diretamente no sistema nervoso central.

Contudo Nessi (2010) evidência que a Massagem Antiestresse possui efeitos terapêuticos e fisiológicos em razão da eliminação dos catabólicos formados nos músculos e diante disso haverá uma na melhora da condutividade elétrica no sistema nervoso central, o que facilitará a circulação do retorno venoso e proporcionará melhor irrigação sanguínea arterial, melhorando consequentemente a depressão, ansiedade, insônia e sintomas emocionais.

Apesar disso, Meneguzzi (2011) contrapõe demonstrando que a Massagem Auyrvédica Abhyanga é uma técnica que utiliza pressão com as mãos, cotovelos, antebraços e pés, visando atingir o sistema muscular, circulatório, linfático, gastointestinal e nervoso. Sabendo disso, a técnica Ayuervédica Abhyanga atua no dosha Vata onde tem em vista o equilíbrio da energia do corpo, harmonização dos chakras, eliminação de toxinas, redução do estresse e ansiedade, melhora da qualidade do sono, melhora da pele e proporcionando equilíbrio físico e metal.

Já Gnatta (2011) salienta que os óleos essenciais são substâncias empregadas com a finalidade de equilibrar as emoções, melhorar o bem-estar físico e mental e que atuam de diversas formas no organismo, podendo ser absorvidas por meio de inalação pelas vias aéreas, por uso tópico ou ingestão. Quando inaladas, uma porcentagem mínima do óleo essencial (OE) ativa o sistema do olfato pelo bulbo e nervos olfativos, que propiciam uma ligação direta com o Sistema Nervoso Central, levando o estímulo ao Sistema Límbico, responsável pelo controle da memória, emoção, sexualidade, impulsos e reações instintivas. O restante da quantidade inalada trafega pelo sistema respiratório e chega à corrente sanguínea. Quando a atuação das moléculas ocorre por via cutânea, o óleo essencial é absorvido e transportado pela circulação sanguínea, sendo conduzido até os órgãos e tecidos do corpo.

Fórum Internacional de Qualidade de Vida e Saúde - Curitiba, 15 de junho de 2019 
Contudo, Rodrigues (2017) demonstra que Massagem Ayurveda é uma técnica que dispõe de movimentos profundos de tração e alongamentos que atuam nos níveis físicos, metais, espirituais, psíquicos e bioenergéticos. Através da descoberta dos doshas que cada um possui, podendo ser o dosha Vata, Pita ou Kapha. No entanto, possui ação de estimular vários pontos bioenergéticos do corpo com o intuito de alinhar o chakra basal, sacral, umbilical, cardíaco, laríngeo, frontal e coronário. Consequentemente causando o desbloqueio da energia vital, melhorando tensões, eliminando toxinas, melhorando o sistema imunológico, circulatório, fazendo aumentar a produção de glóbulos brancos, realinhando os chakras, reequilibrando a energia, levando ao relaxamento, estabiliza o sistema nervoso central, linfático e muscular, provocando o desbloqueio emocional que melhora os sintomas de depressão e estresse.

Mediante disto, Cunico (2017) salienta que a Massagem com Pedras Quentes é uma técnica que utiliza pedras vulcânicas quentes e frias que são ricas em sais mineiras, portanto possuem a capacidade de reter o calor e a energia do corpo e da mente. Todavia, as pedras quando aquecidas estimulam o movimento e reequilíbrio da energia corporal, podendo ter ação nos Chakras, pois a massagem quando realizada se coloca pedras aquecidas e frias sobre os Chacras para obter uma ação bioenergética em todos os pontos do corpo. Sendo então, as pedras aquecidas apropriadas para o tratamento de depressão, ansiedade, relaxamento, dores musculares, estresse e equilíbrio emocional. Já as pedras frias auxiliam na tonificação muscular, revigorando a energia vital, reduzindo inflamações, estimula o sistema nervoso central, reduzindo o estresse, consequentemente são adequadas nos tratamentos de inflamações, edema e flacidez.

No entanto, Alves (2018) salienta que a Candle Massage mais conhecida como Massagem com Velas possui função de aliviar problemas físicos e psicológicos através do toque com óleo vegetal que a vela dispõe, e este toque provoca um estimulo no sistema simpático que informa via aferente para o sistema nervoso central ativando o sistema parassimpático e desativando o sistema simpático fazendo com que o corpo entre em um estado de homeostase, diminuindo a liberação de hormônios do estresse, podendo assim este estimulo trazer fins benéficos como na diminuição da depressão, estresse, fadiga, insônia e ansiedade. Portanto, a técnica com velas proporciona o alivio de tensões musculares, melhora 
na circulação sanguínea, mantem a hidratação da pele por alguns dias, ajuda no equilíbrio energético do corpo, promove o relaxamento muscular e bem-estar.

Embora todos os autores citados anteriormente falem a respeito dos efeitos fisiológicos, emocionais e bioenergéticos ambos não demonstram como esses resultados se manifestam e se correlacionam entre si. Porém, Bear (2017) salienta que os efeitos fisiológicos, emocionais e bioenergéticos geram resultados a níveis fisiológicos sobre a depressão a partir do momento onde ambos se conectam através do sistema nervoso central. Essa conexão se tem através do sistema somatossensorial no qual se permite ter uma sensação agradável ou desagradável que é levada através dos neurônios aferentes que emitem informações realizadas no tecido e órgão para o sistema nervoso central. Sabendo disso, a pele possui muitos axônios que se conectam em vários nervos periféricos e na medula espinhal que fazem o caminho ao sistema nervoso central levado a mensagem do sistema somatossensorial.

Portanto, a partir disso o sistema nervoso central recebe a informação via aferente e após isso se tem uma resposta ao estimulo via neurônios eferentes que emitem a informação do sistema nervoso central as células para os músculos e glândulas, o que causa um efeito fisiológico, emocional e bioenergético através deste processo melhorando efetivamente os sintomas depressivos.

\section{CONCLUSÃO}

O desenvolvimento do presente estudo possibilitou uma análise sobre os efeitos fisiológicos que foram melhora do relaxamento, estimulo no sistema nervoso central, melhora do sistema imunológico, aumento de serotonina, endorfina, melatonina e histamina, já nos efeitos emocionais demonstraram benefícios na melhora da raiva, ansiedade, depressão, estresse, fadiga, insônia, timidez, confusão, humor, medo, culpa, ciúme, debilidade e apatia, e por fim os efeitos bioenergéticos demonstraram benefícios no equilíbrio energético, melhora da energia vital, harmonização dos chakras, equilíbrio físico e metal de diversas massagens que abordaram sobre as causas que provocam os sintomas depressivos em jovens.

De acordo com as pesquisas realizadas verificou-se que em diferentes artigos alguns autores não relacionaram os efeitos das massagens em pessoas com sintomas depressivos. Sendo assim, o foco principal desta pesquisa é enfatizar os Fórum Internacional de Qualidade de Vida e Saúde - Curitiba, 15 de junho de 2019 
benefícios da massagem em jovens com depressão e como essas massagens correlacionam os efeitos fisiológicos, emocionais e bioenergéticos do corpo com o sistema nervoso central.

Portanto concluiu-se que as massagens citadas são benéficas para pessoas com sintomas depressivos e pessoas que não apresentam estes sintomas.

Dada à importância do assunto abordado, abre-se a possibilidade do desenvolvimento de demais pesquisas sobre o assunto, pois devido às pesquisas realizadas neste presente estudo pode-se perceber que há poucos artigos que relacionam os sintomas depressivos com as massagens.

\section{REFERÊNCIAS}

ALVES, A. K.; FERNADES, L. Massagem com a utilização de Velas Quentes. Revista Saúde em Foco, n. 10, p. 168 - 171, 2018.

ANDRADE, R. V.; SILVA. A. F.; MOREIRA, F. N.; SANTOS. H. P. S.; DANTAS, H. F.; ALMEIDA, I. F.; LOBO, L. P. B.; NASCIMENTO, M. A. Atuação dos neurotransmissores na depressão. Rev. Bras. Ciênc. Farm. v. 1, n. 1, p.1 - 4 , Jan./ Mar., 2003.

ANTUNES, S. M.; MONICO, L. S. M. Depressão, ansiedade e stress em doentes deprimidos: Estudo com a EADS-21. INFAD Revista de Psicologia, v.2, n.1, p. 419 - 428, Mar., 2015.

BEAR, M. F.; CONNORS, B. W.; PARADISO, M. A. Neurociências: desvendando o sistema nervoso. 4. Ed. Porto Alegre: Artmed, 2017.

BERTOJA, V. G.; TOKARS, E. Os Benefícios da Massagem Relaxante. 2007. Trabalho de Conclusão de Curso (Especialização em Estética e Imagem Pessoal) Universidade Tuiuti do Paraná, Curitiba: PR, 2007.

CASSAR, M. P. Manual de Massagem Terapêutica: Um guia completo de massaterapia para o estudante e para o terapeuta. 1. Ed. São Paulo: Manole, 2001.

COUTINHO, M. E. M.; GIOVANINI, M.; PAVINI, L. S.; VENTURA, M. T. Aspectos biológicos e psicossociais da depressão relacionado ao gênero feminino. Revista Brasileira de Neurologia e Psiquiatria, v. 19, n. 1, p. 49-57, Jan/Abr., 2015.

CUNICO, I. Massagem com Pedras na Estética. 2017. Trabalho de Conclusão de Curso (Especialização em Estética e Cosmética) - Universidade Tuiuti do Paraná, Curitiba: PR, 2017.

FERREIRA, R. A.; ROSA, W. A. G.; SILVA, V. L. Q.; ALMEIDA, D. A.; OLIVEIRA, I. S. B. Depressão: Ponto de vista e conhecimento de enfermeiros do Programa Saúde 
da Família de São Sebastião do Paraíso. Revista de Iniciação Cientifica da Libertas, v. 5, n.2, p. 143-154, Dez. 2015.

FONSECA, A. A.; COUTINHO, M. P. L.; AZEVEDO, L. W. Representações sociais da depressão em jovens universitários com e sem sintomas para desenvolver a depressão. Psicologia: Reflexão e Critica. [online], v.21, n.3, p.492-498, 2008.

FRITZ, S. Fundamentos da massagem terapêutica. 2. Ed. Barueri: Manole, 2002.

GILLANDERS, A. Guia Completo de Reflexologia. 1. Ed. São Paulo: Pensamento, 2008.

GNATTA, J.R; DORNELLAS, E.V, SILVA, M.J.P. O uso da aromaterapia no alívo da ansiedade. Acta Paulista de Enfermagem, v. 24, n. 2, p. 257-263, 2011.

LARANJEIRA, P. I. C. A relação entre depressão e ideação suicida em jovens adultos: $O$ papel mediador da desesperança e da dor mental. [dissertação]. Évora: Universidade de Évora, Mestrado em Psicologia Área de especialização: Psicologia Clínica e da Saúde; 2015.

LOPEZ, M.R.A; RIBEIRO, J.P; ORES, L.C; JANSEN, K.; SOUZA, L.D.M; PINHEIRO, R.T; SILVA, R.A. Depressão e qualidade de vida em jovens de 18 a 24 anos no sul do Brasil. Revista de Psiquiatria do Rio Grande do Sul. v.33., n.2, p.103-108, 2011.

MENEGUZZI, P.; TURKEWICZ, N. M.; BOBATTO, T. T.; TREML, C. J.; FILHO, F. A. K. Massagem Ayurvédica Abhyanga na Melhora da Qualidade de Vida, Dor e Depressão em Portadores de Fibromialgia. Revista Brasileira de Terapias e Saúde, v. 1, n. 2, p. 65 - 74, Jul., 2011.

$\mathrm{NESSI}$ A. Massagem antiestresse: Uma abordagem teórica e pratica para o bem estar. 5. Ed. São Paulo: Phorte, 2010.

RÉGIS, B. N., ARAÚJO, R. L. R., SOUZA, V. G., NETO, N. A. S., NODARI, N. L., HAYASIDA, N. M. A. Ansiedade, depressão e doença cardiovascular em jovens adultos: uma revisão da literatura. Revista Saúde e Desenvolvimento Humano, v. 4, n.1, p. 91-100. Mai, 2016.

RODRIGUES, A. L. S. Ayurveda Massage: novo paradigma na busca da saúde. 2017. Trabalho de Conclusão de Curso (Especialização em Estética e Imagem Pessoal) - Universidade Tuiuti do Paraná, Curitiba: PR, 2017.

SEUBERT, F.; VERONESE; L. A massagem terapêutica auxiliando na prevenção e tratamento das doenças físicas e psicológicas. In: Encontro Paranaense, Congresso Brasileiro, Convenção Brasil/Latino-América, XIII, VIII, II, 2008. Anais. Curitiba: Centro Reichiano, 2008.

WHO, World Health Organization. Young People's Health - a Challenge for Society. Report of a WHO Study Group on Young People and Health for All. Technical Report Series 731. Geneva: WHO, 1986. 
WHO. Journée internationale de la jeunesse : des espaces sécurisés pour des adolescents en bonne santé. Disponível em: http://www.euro.who.int/fr/healthtopics/Life-stages/pages/news/news/2018/08/international-youth-day-safe-spacesfor-healthy-adolescents. Acessado em: 28, mar, 2019. 